\title{
Oportunidad de la sistematización de la vigilancia de Dengue en Paraguay, su estacionalidad y las características de los casos en menores de 15 años, 2010-2012
}

\author{
Timeliness of the Dengue Vigilance System in Paraguay: \\ Seasonal Effects and Characteristics of Cases in Children \\ under Age 15, 2010-2012
}

\author{
Mara Muñoz $^{(1)}$, Celia Martínez de Cuellar ${ }^{(2)}$, Dalva Assis ${ }^{(1)}$
}

\section{RESUMEN}

Introducción: El flujo de información de la vigilancia de dengue en Paraguay va desde el nivel primario hasta niveles nacionales, debiendo cumplir plazos preestablecidos. Objetivos: Definir la estacionalidad, describir epidemiológicamente los casos en menores de 15 años y la oportunidad de sistematización de datos. Materiales y Métodos: Estudio descriptivo retrospectivo, utilizando las notificaciones de dengue entre 2010 y 2012. Se evaluaron las oportunidades de acceso a los servicios, llenado de fichas, registro, remisión y digitación. Resultados: Se registraron 112.944 notificaciones, 21.557 en 2010, 51.763 en 2011 y 39.624 en 2012, el primer semestre de cada año presentó $90 \%$ de los casos. El $21 \%$ correspondió a menores de 15 años, sin predominancia de sexo, con una media de 8,6 años. La oportunidad de acceso a los servicios fue en 2010 y 2011 de dos días y tres días en 2012. La oportunidad de llenado de fichas fue en 2010 un día, dos en 2011 y en 2012 tres días. La oportunidad de registro fue en 2010 de 24 días, 22 en 2011 y en 2012 seis días. La oportunidad de remisión de fichas fue de seis días en 2010, 22 en 2011, cinco días en 2012; la oportunidad de digitación nueve días en 2010, dos en 2011 y un día en el 2012. Conclusiones: Existieron más notificaciones en el primer semestre señalando estacionalidad de la enfermedad. El llenado de fichas es oportuno, pero su remisión inoportuna, lo que puede afectar el control del foco.

Palabras clave: Dengue, notificación de enfermedad, vigilancia epidemiológica, Paraguay.

\section{ABSTRACT}

Introduction: Information flow for surveillance of dengue in Paraguay reaches from primary facilities to the national level, and includes prescribed reporting deadlines. Objectives: To determine seasonal effects, describe cases in children under age 15 epidemiologically, and quantify timeliness of entry into data systems. Materials and Methods: We conducted a retrospective descriptive study using Dengue Reports from 2010 through 2012. Timeliness of access to service, case-report completion, recording, transmittal, and data entry were assessed. Results: A total of 112,944 case reports were recorded; 21,557 in 2010, 51,763 in 2011, and 39,624 in 2012, with $90 \%$ of cases recorded in the first six months of the year. Some $21 \%$ of cases were in patients under age 15 (mean: 8.6 years), without differences by sex. Time to access-to-services was 2-3 days in 20102011, and 3 days in 2012. Time to case report completion was 1 day in 2010, 2 days in 2011, and 3 days in 2012. Time to recording was 24 days in 2010, 22 days in 2011, and 6 days in 2012. Time to transmittal of case-reports was 6 days in 2010, 22 days in 2011, and 5 days in 2012, while time to data entry was 9 days in 2010, 2 days in 2011, and 1 day in 2012. Conclusions: A majority of reports occur in the first six months of the year, indicating the seasonal nature of the disease. The completion of case reports is timely, but their transmittal untimely, which can affect control of disease transmission sites.

Keywords: Dengue; disease notification; surveillance, epidemiology; Paraguay.

\section{Programa de Maestría en Epidemiologia de Campo. Asunción, Paraguay.}

2. Dirección General de Vigilancia de la Salud, Ministerio de Salud Pública y Bienestar Social. Asunción, Paraguay.

Correspondencia: Lic. Mara Muñoz.E-mail:mara-munoz@hotmail.com

Recibido: 4/06/2014; Aceptado: 16/10/2014.

http://dx.doi.org/10.18004/ped.2015.abril.11-16

Los autores declaran que no existen conflictos de interés en el presente estudio. 
El dengue es una infección que posee un espectro que varía desde una forma asintomática hasta cuadros de hemorragia y choque, pudiendo evolucionar inclusive al óbito ${ }^{(1)}$. Es la arbovirosis más importante que afecta a los seres humanos y se presenta principalmente en los países tropicales, donde las condiciones ambientales favorecen el desarrollo del vector ${ }^{(2)}$. En menos de 20 años, la región se ha transformado de hipoendémica a hiperendémica en países con características ambientales tropicales favorables del continente americano, con varios serotipos del virus en circulación (Denv-1, Denv-2, Denv-3 y Denv-4) $)^{(3-5)}$.

Con la ausencia de una vacuna para combatir la enfermedad, las medidas de control incluyen acciones de vigilancia epidemiológica y entomológica. El primero tiene como objetivo monitorear la evolución temporal de la incidencia de casos en el espacio geográfico específico y confrontar con la incidencia de infestación por el vector, organizar discusiones conjuntas con los equipos de control de vectores y la asistencia, para discutir los resultados de las investigaciones de las muertes y otras medidas del plan de contingencia a niveles jerárquicos cuando hay riesgo de epidemias y vigilar la circulación del virus para detectar precozmente la introducción de un nuevo serotipo en la ciudad ${ }^{(6,7)}$.

La vigilancia epidemiológica y la vigilancia entomológica juegan un rol capital en la determinación de los riesgos epidemiológicos para la ocurrencia de brotes o epidemias, ambos conducen las estrategias de las intervenciones con información útil para direccionar las acciones comunitarias de los gobiernos locales para el mejoramiento de la situación ambiental ${ }^{(7)}$. El éxito de estas actividades debe estar vinculado a la notificación de los casos y su remisión, pensando en la oportunidad de un corte de transmisión, debiendo ésta realizarse en forma y en tiempo ${ }^{(8)}$. Así como lo establece la resolución ministerial S.G. № 11/85 que reglamentan los artículos $28^{\circ}$, referente a la notificación obligatoria de eventos, el artículo 37ํㅜㅇ relacionado con eventos sujetos a vigilancia epidemiológica y 33ํㅡㄹ la Ley № 836/80.

De igual manera el Código Sanitario emitido el $13 / 01 / 2005$, afirma que el dengue es una enfermedad de notificación obligatoria, en forma nominal, por ficha individual dentro de las 24 horas de la sospecha clínica, al siguiente nivel, todos los días de la semana a cualquier hora. Entonces el proceso de vigilancia del dengue inicia con la notificación a través de una ficha individual que diligencia un profesional de salud en la institución donde se identificó el caso. El flujo de información va entonces de los sistemas primarios a las direcciones nacionales, debiendo cumplir plazos en cada uno de ellos ${ }^{(9)}$.

En la guía para la evaluación de los sistemas de vigilancia en salud pública del Centers for Disease Control and Prevention, dice que la oportunidad es la puntualidad o velocidad con que el personal encargado de ejecutar las acciones en cada nivel o paso del sistema de vigilancia en salud pública, tarda en hacerlo. Entonces, desde el punto de vista de la vigilancia del dengue la oportunidad es el tiempo que se demora en notificar la enfermedad desde los centros primarios a las unidades epidemiológicas regional hasta el nivel central y su procesamiento para generar información ${ }^{(10)}$.

El llenado de las fichas individuales es un acto médico, donde los campos deben ser consignados en el momento de la consulta, en los centros asistenciales en forma manual, éstos registros son recolectados por los encargados de la vigilancia de cada servicio para ser posteriormente remitidos al siguiente nivel, ya sea por fax, internet o currier, para la digitalización de los datos y su posterior análisis en el nivel central.

Consideramos que el dengue es una enfermedad epidémica cuya frecuencia en la población es alta, en esta investigación se analizan los siguientes objetivos: definir la estacionalidad de la enfermedad, describir epidemiológicamente los casos en menores de 15 años y sobre todo describir la oportunidad de las acciones de vigilancia en cuanto a la sistematización de los datos, por último proponer recomendaciones para el fortalecimiento del control de dengue en Paraguay.

\section{MATERIALES Y MÉTODOS}

Estudio descriptivo retrospectivo de tipo corte 
transversal teniendo a la República de Paraguay como población de estudio, de acuerdo a la distribución de los casos notificados a la Dirección General de Vigilancia de la Salud (DGVS) dependiente del Ministerio de Salud y Bienestar Social. Se utilizó como fuente de datos las planillas electrónicas donde están cargadas las notificaciones de dengue de los años 2010, 2011 y 2012.

Se incluyeron en este estudio las variables: edad, sexo, departamento de residencia y las fechas que tenían que ver con las oportunidades de registro de la información (fecha de inicio de la fiebre, fecha de consulta, fecha de notificación, fecha de mesa de entrada a la DGVS y fecha de digitación). Fueron incluidos los casos clasificados como sospechosos y confirmados.

Fueron excluidos el $7 \%$ de los registros por presentar datos incoherentes y el $44 \%$ de los registros por mostrar datos incompletos. Se describieron las siguientes cinco oportunidades:

Las oportunidades fueron expresadas en número de días, de la siguiente manera:

\section{Oportunidad de los casos para acceder a los} servicios: Fecha de consulta - fecha de inicio de la fiebre; esta oportunidad sirvió para conocer el tiempo que la población tarda en llegar a un centro asistencial, donde se juzgó la percepción de riesgo de la población en relación a la enfermedad.

2. Oportunidad de llenado de fichas: Fecha de notificación o llenado de fichas - fecha de consulta; el tiempo que demora el personal de salud en notificar, que permitió juzgar la percepción de los profesionales de salud en relación a la notificación obligatoria de la enfermedad para la toma de acciones.

3. Oportunidad de registro: Fecha de digitación en el sistema informatizado - fecha de notificación; es el tiempo total empleado, desde el llenado de la ficha en el servicio de salud hasta el registro en una base de datos, lo que permitió juzgar el tiempo utilizado para tener datos analizables y proponer acciones de prevención y control. Esta oportunidad fue desglosada en dos etapas, la primera desde el llenado de las fichas en los servicios hasta la llegada a la DGVS, y la otra desde la llegada hasta la introducción de los datos en una base informatizada, a fin de determinar en cuál de las instancias del proceso de registro de la información hubieron retrasos, atendiendo a que el dengue es una enfermedad de notificación obligatoria e inmediata.

3.a) Oportunidad de remisión de fichas: Fecha de entrada a la DGVS - fecha de notificación; esta oportunidad permitió conocer el tiempo empleado desde el llenado de la fichas hasta la remisión al sistema de vigilancia, así juzgamos si el personal está sensibilizado con la inmediatez de la notificación.

3.b) Oportunidad de digitación: Fecha de digitación - fecha de entrada a la DGVS; esta oportunidad permitió hallar el tiempo empleado desde la entrada de la ficha a la DGVS hasta su posterior digitación, con el propósito de conocer el tiempo empleado para transformar la información encontrada en papel en un formato electrónico.

Los resultados se presentaron teniendo en cuenta los promedios de intervalos de días para cada oportunidad, mostrando los rangos mínimo y máximo, y se calculó para cada una el cuartil 75. Los análisis fueron realizados en Microsoft Excel ${ }^{\circledR}$.

Considerando el aspecto ético se utilizaron datos secundarios manteniendo el sigilo de los pacientes, manejando datos solamente para obtener los objetivos propuestos.

\section{RESULTADOS}

\section{Distribución temporal de los casos de dengue}

Durante el periodo de estudio, se registraron 112.944 notificaciones por dengue, en el año 2010 hubieron 21.557 notificaciones, en el 2011 fueron 51.763 y en el año 2012 fueron 39.624, de estas se confirmaron como dengue el 78\% (87.956/112.944), se descartaron $10 \%(11.716 / 112.944)$ y quedaron como sospechosos 12\% (13.112/112.944) de los casos.

$\mathrm{Al}$ analizar la ocurrencia de casos según la estacionalidad, en los tres años, el 90\% (100.580/112.944) de las notificaciones se presentaron en el primer semestre del año, reduciéndose notablemente en los meses de julio a diciembre, sin embargo no se observa un corte en la transmisión (Figura 1). 


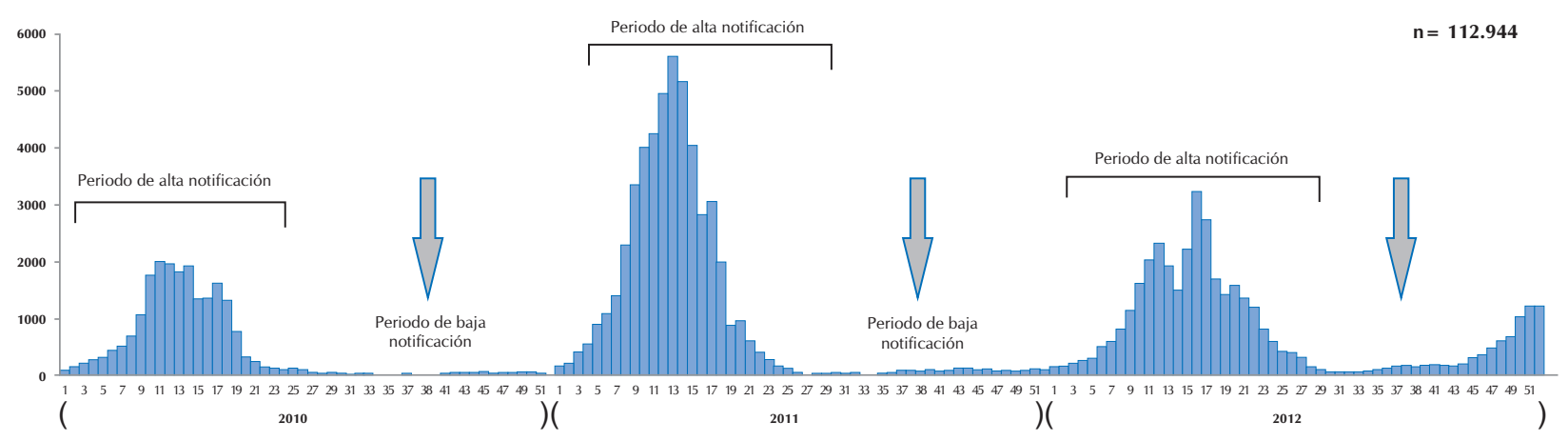

Figura 1. Notificaciones de dengue por semana correspondiente a los años 2010 al 2012, Paraguay.

Comparando estos periodos encontramos que en el primer semestre de los tres años evaluados, el $82 \%$ de las notificaciones fueron confirmadas para dengue y en el segundo semestre un $5 \%$ (Tabla 1).

Tabla 1. Distribución de notificaciones de dengue según clasificación y periodo de presentación 20102012, Paraguay.

\begin{tabular}{|c|c|c|c|c|}
\hline \multirow{3}{*}{$\begin{array}{l}\text { Clasificación } \\
\text { de los casos }\end{array}$} & \multicolumn{4}{|c|}{ Notificaciones por Semestres } \\
\hline & \multicolumn{2}{|c|}{$1^{\circ}$ Semestre $(n=101.068)$} & \multicolumn{2}{|c|}{$2^{\circ}$ Semestre $(n=8.192$} \\
\hline & $\mathrm{n}$ & $\%$ & $\mathrm{n}$ & $\%$ \\
\hline Confirmados & 82.932 & 82 & 5.240 & 5 \\
\hline Sospechosos & 9.944 & 10 & 2.952 & 3 \\
\hline
\end{tabular}

\section{Distribución en los menores de 15 años}

En el periodo de estudio el 21\% (21.223/99.557) de los casos correspondieron a niños menores de 15 años, en el año 2010 representaron el 18\% (3.545/19.552), en el 2011 el 23\% (10.556/45.294) y en el 2012 el 28\% (9.835/34.708) del total de notificaciones para todas las edades. No existe predilección por el sexo en éste grupo etario. De acuerdo al lugar de residencia, el área metropolitana compuesta por el departamento Central y la Capital presentó el 76\% (17.119/22.633) de las notificaciones en éste grupo de edad.

\section{Oportunidad del sistema de vigilancia}

En el año 2010, se observó en promedio de días que la oportunidad de acceso a servicios de salud fue de tres días. La oportunidad de llenado de fichas fue de un día, la oportunidad de registro fue de 24 días, la oportunidad de remisión de fichas fue de seis días y la oportunidad de digitación nueve días.

En el año 2011, se observó en promedio de días que la oportunidad de acceso a servicios de salud fue tres días. La oportunidad de llenado de fichas fue de dos días, de registro 22 días, de remisión de fichas 22 días y de digitación dos días.
En el año 2012, se observó en promedio de días que la oportunidad de acceso a servicios de salud fue dos días. La oportunidad de llenado de fichas fue de tres días, de registro fue de seis días, de remisión de fichas cuatro días y de digitación de dos días (Tabla 2).

Tabla 2: Resultado de las oportunidades en días de los años 2010, 2011 y 2012, Paraguay.

\begin{tabular}{|c|c|c|c|c|c|c|c|c|c|}
\hline \multirow{2}{*}{ Tipo de oportunidad } & \multicolumn{3}{|c|}{2010} & \multicolumn{3}{|c|}{2011} & \multicolumn{3}{|c|}{2012} \\
\hline & $\mathrm{m}$ & rango & $q$ & $\mathrm{~m}$ & rango & $q$ & $\mathrm{~m}$ & rango & $q$ \\
\hline $\begin{array}{l}\text { Oportunidad de acceso } \\
\text { a los servicios }\end{array}$ & 3 & $(0-217)$ & 3 & 3 & $(0-217)$ & 3 & 2 & $(0-217)$ & 3 \\
\hline $\begin{array}{l}\text { Oportunidad de llenado } \\
\text { de fichas }\end{array}$ & 1 & $(0-309)$ & 1 & 2 & $(0-217)$ & 2 & 3 & $(0-217)$ & 3 \\
\hline Oportunidad de registro & 24 & $(0-67)$ & 24 & 22 & $(0-217)$ & 28 & 6 & $(0-217)$ & 6 \\
\hline $\begin{array}{l}\text { Oportunidad de remisión } \\
\text { de fichas }\end{array}$ & 6 & $(0-366)$ & 5 & 22 & $(0-217)$ & 0 & 4 & $(0-217)$ & 5 \\
\hline $\begin{array}{l}\text { Oportunidad de } \\
\text { digitación }\end{array}$ & 9 & $(0-106$ & 7 & 2 & $(0-217)$ & 0 & 2 & $(0-217)$ & 2 \\
\hline
\end{tabular}

\section{DISCUSIÓN}

El dengue en Paraguay tiene un comportamiento estacional, como se ha descrito en países de Centro América donde el dengue presenta un patrón estacional con incremento de la incidencia durante la estación lluviosa ${ }^{(11,12)}$, donde el periodo de mayor notificación corresponde al primer semestre del año, entre los meses de enero a junio. En los meses siguientes los reportes de casos se reducen al mínimo. Esta época del año debería ser encarada como punto clave de vigilancia, pues es importante una buena sensibilidad del sistema para la detección de posibles nuevos casos. Considerando que en el periodo de estudio no existió corte de circulación viral, razonamos entonces que el dengue se presenta en el país de forma endémica, es decir, debe ser considerada enfermedad endemo-epidémica ${ }^{(13)}$.

Las notificaciones de dengue en el grupo de edad menores de 15 años fueron aumentando progresiva- 
mente, a través de los tres años. Además como en los otros grupos de edad no se encontraron diferencias en la presentación según el género.

En cuanto las oportunidades, punto central de este trabajo vemos con respecto a la percepción de la enfermedad en la población, ésta acude al servicio al segundo o tercer día de iniciado los síntomas, lo que traduce un conocimiento hacia la posible gravedad de la enfermedad o por las molestias ocasionadas en la forma de presentación de los síntomas.

El llenado de las fichas en los servicios de salud es oportuno, creemos que el personal de blanco acepta notificar la enfermedad en una ficha epidemiológi$\mathrm{ca}$, pues reconoce la obligatoriedad de esta notificación. Sin embargo cuando hablamos de la oportunidad de remisión de las fichas epidemiológicas al sistema de vigilancia, la misma no es oportuna, lo que traduce que el personal encargado de dicha tarea, no comprende que el dengue además de ser una enfermedad de notificación obligatoria es también de notificación inmediata, esto indudablemente afecta al control del foco principalmente en épocas de epidemia.

Con respecto a las oportunidades de remisión y notificación de fichas, fue mejorando en el último año, así en el año 2010 la debilidad se encontraba en la digitación de las fichas y en el año 2011 en la remisión, en el año 2012 el tiempo se redujo, pero para que el sistema de vigilancia sea oportuna la remisión de fichas debe ser inmediata.

Es imprescindible contar con un sistema de información ágil atendiendo al problema que acarrea el dengue para la salud pública. La rapidez en la detección constituye la característica más importante para el funcionamiento de estos sistemas y muchos de ellos funcionan en tiempo real, a la vez que se recibe la información de las diversas fuentes se preparan los mecanismos para las alertas inmediatas. Esto difícilmente puede lograrse con la información que rutinariamente registran los sistemas de vigilancia convencionales, los cuales generalmente dependen del reporte manual de los casos desde que el médico u otro personal de salud atienden al paciente, hasta que otro personal procesa las estadísticas. Esta sería la ventaja fundamental de la utilización de los sistemas automatizados para la detección de alertas ${ }^{(14,15)}$.

\section{CONCLUSIONES}

El dengue tiene un comportamiento estacional con aumento de casos en las estaciones de verano otoño. Por lo que es conveniente dividir el año en cuanto a las acciones en dos periodos; el estacional y el pos estacional.

La incidencia de dengue en la población pediátrica está en aumento. Hay que tomar recaudos en este grupo, pues pueden causar en segundos episodios o manifestaciones más graves por la infección con serotipos distintos.

Es imperativo informatizar los sistemas de vigilancia epidemiológica para que la remisión de datos se efectúe en forma oportuna, y se cumpla con los objetivos de la vigilancia.

Se observó que la población tiene percepción de riesgo frente al dengue. El personal de los servicios reconoce que el dengue es una enfermedad de notificación obligatoria, sin embargo hay problemas en la comprensión de que es además una enfermedad de notificación inmediata, vale decir dentro de las 24 horas de sospechar el caso. Sin embargo se observó que la sistematización de los datos fue mejorando en el último año.

Con los resultados obtenidos en este análisis, se pudieron identificar debilidades importantes en el sistema de vigilancia y que el retraso en las notificaciones pudo haber influido de alguna manera en la magnitud de las epidemias, al retrasar las acciones de bloqueo de casos y toma oportuna de decisiones. Se recomienda insistir en la capacitación al personal de los servicios en la correcta y oportuna notificación de casos de dengue, además de planificar a mediano y largo plazo el fortalecimiento de la red de unidades epidemiológicas regionales, para que local y regionalmente se empoderen de las acciones a ser realizadas dentro de los procesos de vigilancia.

\section{AGRADECIMIENTOS}

Agradecemos a la Dra. Malvina Páez por su colaboración en la corrección de la escritura y a la Lic. Dora Ramírez por la traducción del resumen al inglés. 


\section{REFERENCIAS}

1. Figueiredo LTM, Fonseca BAL. Dengue. In: Veronesi R, Focaccia R. Tratado de Infectologia. $2^{\underline{a}}$ ed. Rio de Janeiro: Atheneu;2002. p. 204-17.

2. Mackenzie JS, Gubler DJ, Petersen LR. Emerging flaviviruses: the spread and resurgence of Japanese encephalitis, West Nile and dengue viruses. Nat Med. 2004;10:S98-109.

3. Isturiz RE, Gubler DJ, Brea del Castillo J. Dengue and dengue hemorrhagic fever in Latin America and the Caribbean. Infect Dis Clin North Am. 2000;14:121-40.

4. Branch SL, Levett PN. Evaluation of four methods for detection of immunoglobulin $\mathrm{M}$ antibodies to dengue virus. Clin Diagn Lab Immunol. 1999;6:555-57.

5. Cruz Cubas A, Rolland-Burger L. El virus del dengue. Diagnóstico [Internet]. 2002 [citado 2004 ene 20];41(4):16572. Disponible en: http://www.fihu diagnostico.org.pe/revista/numeros/2002/julago02/165172.html

6. Tauil, PL. Urbanização e ecologia do dengue. Cad Saúde Pública [Internet]. 2001 [citado 2010 agost 25];17(Supp1.):S99-S102. Disponible en: http://www.scielo.br/scielo.php?script=sci_arttext\&pid=S 0102-311X2001000700018

7. Ministério da Saúde. Diretrizes nacionais para prevenção e controle de epidemias de dengue. Brasília, DF: Ministério da Saúde; 2009.

8. Baly A, Toledo ME, Vanlerberghe V, Ceballos E, Reyes A, Sanchez I, Carvajal M, Maso R, La Rosa M, Denis O, Boelaert M, Van der Stuyft P. Cost-effectiveness of a community-based approach intertwined with a vertical
Aedes control program. Am J Trop Med Hyg. 2009;81(1):88-93.

9. Instituto Nacional de Salud de Colombia. Vigilancia y control en salud pública, SIVIGILA 2008: protocolo de vigilancia de dengue. Colombia: Instituto Nacional de Salud; 2007.

10. Guidelines Working Group. Updated guidelines for evaluating public health surveillance systems. MMWR Recommendations and Reports [Internet]. 2001 [citado 2014 agot 26];50(RR13):1-35. Disponible en: http://www.cdc.gov/mmwr/preview/mmwrhtml/rr5013a 1.htm

11. Siqueira J, Turchi C, Coelho G, da Rocha A. Dengue and dengue hemorrhagic fever, Brazil, 1981-2002. Emer Infec Dis. 2005;11:48-53.

12. Perez R. Dengue surveillance, United States, 19861992. MMWR CDC Surveill Sum. 1994;43:7-19.

13. Guzmán Tirado M, Kourí Flores G, Bravo Gonzalez Y. La emergencia de la fiebre hemorrágica del dengue en las Américas: reemergencia del dengue. Rev Cubana Med Trop. 1999;51(1):5-13.

14. Wagner MM, Espino J, Tsui F-C, Harrison L, Pasculle W. Real-time detection of disease outbreaks. Technical Report [Internet]. 2000 [citado 2014 agost 26]. Disponible en: http://citeseerx.ist.psu.edu/viewdoc/download?doi= 10.1.1.117.2410\&rep=rep1\&type=pdf

15. Dato V, Wagner MM, Fapohunda A. How outbreaks of infectious disease are detected: a review of surveillance systems and outbreaks. Public Health Reports. 2004;119(5):464-71. 